\title{
Genetic parameters for natural antibodies and associations with specific antibody and mastitis in Canadian Holsteins
}

\author{
K. A. Thompson-Crispi, ${ }^{* 1}$ F. Miglior, † \\ *Department of Pathobiology, University of Guelph, Guelph, Ontario, N1G 2W1, Canada \\ †Guelph Food Research Center, Agriculture and Agri-Food Canada, Guelph, Ontario, N1G 5C9, Canada \\ ‡Canadian Dairy Network, Guelph, Ontario, N1K 1E5, Canada
}

\section{ABSTRACT}

The objectives of this study were to evaluate (1) natural antibodies (NAb) in Canadian Holstein cows, (2) genetic parameters and associations between NAb and specific antibody (SpAb), and (3) the association of $\mathrm{NAb}$ with clinical mastitis and differences in incidence rates of clinical mastitis (IRCM) among cows classified as high, average, or low responders for NAb. Natural antibodies (IgG and IgM) to keyhole limpet hemocyanin and SpAb to a type 2 test antigen were measured on 451 Holsteins from 41 herds across Canada. A series of uni- and tri-variate linear animal models were used to estimate genetic parameters and breeding values for $\mathrm{NAb}$ and SpAb. The models included the fixed effects of parity and stage of lactation and the random effects of herd-technician, animal, and residual. Using estimated breeding values for NAb, cows were classified as high, average, or low responders and phenotypic associations with the IRCM were investigated and a logistic regression performed. The estimated heritability was 0.27 for $\mathrm{SpAb}$, and was 0.32 and 0.18 for NAb of the IgG and IgM isotypes, respectively. No significant genetic correlations were found between $\mathrm{SpAb}$ and NAb. Although no significant differences in the IRCM were found when cows were classified based on NAb IgG, cows classified as high responders for NAb IgM tended to have a lower IRCM compared with other cows. Immunoglobulin-M was associated with a decreased risk of clinical mastitis (odds ratio $=0.958$ ). Results of this study suggest the potential to use NAb IgM as an additional tool to select for disease resistance in cattle, but results need to be validated with a larger sample size.

Key words: natural antibodies, immune response, mastitis, genetic parameter

Received July 7, 2012.

Accepted February 22, 2013.

${ }^{1}$ Corresponding author: kthomp02@uoguelph.ca

\section{INTRODUCTION}

A robust and balanced immune system of the dairy cow is vital for protection against economically important diseases. Holstein cows with superior or high antibody- (AMIR) and cell-mediated immune responses (CMIR) have been shown to have a lower occurrence of diseases, including mastitis, metritis, retained placenta, and displaced abomasum, and are less likely to be seropositive for Mycobacterium avium ssp. paratuberculosis compared with average- and low-immune responding cows (Wagter et al., 2000; Pinedo et al., 2009; Thompson-Crispi et al., 2012a). The adaptive immune response traits, AMIR and CMIR, predominate in protection against extracellular and intracellular pathogens, respectively, and both are therefore required for broad based disease resistance. Both AMIR and CMIR have been demonstrated to be heritable ( 0.29 and 0.19 , respectively), and their inclusion in breeding indices has been suggested to improve inherent animal health and decrease the incidence of disease in the dairy industry (Mallard et al., 2011; Thompson-Crispi et al., 2012b). Previous studies in numerous herds have shown specific antibody (SpAb) to be a reliable predictor of enhanced dairy cattle health (Wagter et al., 2000; Mallard et al., 2011; Thompson-Crispi et al., 2012a), and this association has recently been confirmed in a national immune response study across Canada. Cows classified as high for SpAb had a lower incidence rate and less severe clinical mastitis compared with other cows in the herd (Thompson-Crispi et al., 2013).

Natural antibodies (NAb), on the other hand, are antibodies that circulate in normal individuals in the absence of exogenous antigenic stimulation, and have been considered as a humoral component of the innate immune system (Baumgarth et al., 2005). Natural antibodies are predominantly of the IgM isotype, and to a lesser extent IgG and IgA (Kohler et al., 2003), and are typically polyreactive to conserved structures such as nucleic acid, carbohydrates, and phospholipids (Boes, 2000). Natural antibodies are produced by B-1 cells, a long-lived, self-renewing B cell subset that differs from 
conventional B-2 cells in their ontogeny and localization in peritoneal and pleural cavities (Baumgarth, 2011). The B-1 cells lack terminal deoxynucleotidyl transferase activity early in ontogeny contributing to a more limited set of gene rearrangements and restricted $\mathrm{NAb}$ repertoire than antibodies produced by conventional B cells (Boes, 2000). Natural antibodies provide protection against infection by direct neutralization, activation of the complement system, and formation of antigen-antibody complexes leading to pathogen elimination in the spleen, thereby enhancing specific immune responses in secondary lymphoid organs (Ochsenbein and Zinkernagel, 2000).

Keyhole limpet hemocyanin $(\mathbf{K L H})$ is a high-molecular mass metalloprotein derived from the giant keyhole limpet Megathura crenulata (Harris and Markl, 1999) that has remarkable immunostimulatory properties. As it is derived from the ocean, livestock species are naive to KLH, making it a good antigen to measure NAb. In dairy cattle, NAb-binding KLH have been found in both milk and serum, and their measurements have been shown to be repeatable over time (Ploegaert et al., 2011). The across-herd heritability of bovine NAb to KLH has been estimated to be $0.36(\mathrm{SE}=0.08)$, and NAb have been suggested as potential immune parameters for disease resistance in dairy cows (Ploegaert et al., 2010). Previous studies in poultry have found NAb to be positively correlated with survival and health status (Star et al., 2007). However, a direct correlation of NAb and disease in dairy cattle has yet to be demonstrated, as has been shown in multiple studies for SpAb (Mallard et al., 1997; Wagter et al., 2000; Thompson-Crispi et al., 2012a). Therefore, the objectives of this study were to (1) evaluate NAb of the IgG and $\operatorname{IgM}$ isotypes in serum to KLH of 451 Holstein cows across Canada that have previously been immune response phenotyped for $\mathrm{SpAb},(2)$ estimate the genetic parameters and associations between NAb and SpAb, and (3) determine the association with NAb and clinical mastitis and evaluate phenotypic differences in the incidence rate of clinical mastitis between cows classified as high, average, or low immune responders for NAb.

\section{MATERIALS AND METHODS}

\section{Animals}

Immune response profiles of 451 lactating Holsteins, outside the peripartum period, from 41 herds across Canada were evaluated (Thompson-Crispi and Mallard, 2012) in collaboration with the Canadian Bovine Mastitis Research Network (CBMRN). Lactating cows enrolled in the longitudinal incidence-density mastitis sampling for the CBMRN were also immune response tested (Reyher et al., 2011). Ten cows expected to remain in the herd for at least 2 mo were chosen at random along with the 5 cows that had most recently calved. Immune responses were tested between July 2007 and August 2008. Distribution by parity was as follows: $\mathrm{n}=141$ in parity $1, \mathrm{n}=134$ in parity $2, \mathrm{n}$ $=77$ in parity 3 , and $n=99$ in parity 4 or higher. All experimental procedures were approved by the Animal Care Committee of the University of Guelph under guidelines of the Canadian Council of Animal Care.

\section{Immunization Protocol for Specific Antibody}

Cows at least 28 DIM were immunized to stimulate AMIR, as described and reported previously (Thompson-Crispi et al., 2012b). Briefly, the primary antibody to a type 2 test antigen, hen egg white lysozyme, was used as an indicator of AMIR. On d 0 cows received an intramuscular injection of $0.5 \mathrm{mg}$ of hen egg white lysozyme (Sigma-Aldrich Canada Ltd., Oakville, Canada) and $0.5 \mathrm{mg}$ of Quil-A adjuvant (Cedarlane Laboratories, Hornby, Canada) dissolved in $1 \mathrm{~mL}$ of PBS (pH 7.4).

\section{Specific Antibody}

Blood was collected on d 0 and 14 of the immunization protocol to evaluate the specific serum antibody of the IgG1 isotype to the type 2 test antigen by a modified ELISA as described and reported previously (Thompson-Crispi et al., 2012b).

\section{Natural Antibody Positive Control}

Three cows were immunized with $1.0 \mathrm{mg}$ of Megathura crenulata-derived KLH from MP Biomedicals (Solon, $\mathrm{OH})$ and $0.5 \mathrm{mg}$ of Quil-A adjuvant (Cedarlane Laboratories Ltd.) dissolved in $1 \mathrm{~mL}$ of PBS ( $\mathrm{pH}$ 7.4). Using a 22-gauge needle, a 1.0-mL injection was administered intramuscularly in the rump. Cows were boosted $14 \mathrm{~d}$ later to achieve maximum antibody response to $\mathrm{KLH}$ on d 21. Serum was collected at d 0,14, and 21, and the cow with the highest serum IgG and IgM to KLH on d 21 was used as the positive control.

\section{Natural Antibodies}

Serum from d 0 of the immunization protocol was used to evaluate natural antibody to KLH by a modified ELISA. The negative control was fetal calf serum. Flat bottomed 96-well polystyrene plates were coated with $100 \mu \mathrm{L} /$ well of $1 \mu \mathrm{g}$ of KLH dissolved in $1 \mathrm{~mL}$ of carbonate-bicarbonate buffer ( $\mathrm{pH} 9.6)$ and incubated at $4^{\circ} \mathrm{C}$ for $24 \mathrm{~h}$. Plates were washed with PBS and $0.05 \%$ 
Tween 20 (Sigma-Aldrich Canada Ltd.; wash buffer pH 7.4) 3 times and blocked with PBS, $3 \%$ Tween 20 , $1.5 \%$ BSA, and $1.5 \%$ fetal calf serum for $1 \mathrm{~h}$ at room temperature (RT) and then washed again 3 times. Four serial dilutions (1:4) starting at 1/40 of samples in wash buffer were added to the plate and incubated for $2 \mathrm{~h}$ at RT. Plates were washed 5 times and a secondary antibody, either 1:1,000 monoclonal anti-bovine IgG from mouse ascites fluid (Sigma-Aldrich, St. Louis, MO) or 1:5,000 anti-bovine $\operatorname{IgM}$ produced in sheep (Bethyl Laboratories, Montgomery, TX) conjugated to alkaline phosphatase, was dissolved in Tris-Tween buffer with $0.05 \%$ Tween 20 (pH 7.4) and incubated at RT for $1 \mathrm{~h}$. All wash steps were performed with the Elx405 Auto Plate Washer (Biotek Instruments Inc., Winooski, VT). p-Nitrophenyl phosphate substrate system (Sigma-Aldrich Canada Ltd.) was added and incubated for approximately $50 \mathrm{~min}$ at RT and optical density (OD) values at $405 \mathrm{~nm}$ were obtained using the EL808 (BioTek Instruments Inc.) plate reader. Data were collected with Gen5 Software (BioTek Instruments Inc.); OD values were corrected to the rolling mean of the positive controls for each plate as described previously (Heriazon et al., 2009) to account for day and plate variation. The dilutions of the corrected $\mathrm{OD}$ values were summed and duplicates averaged for statistical analysis.

\section{Mastitis Sampling}

Milk samples were taken as described previously for the CBMRN herds (Reyher et al., 2011). Briefly, farmers sampled cows identified as having abnormal milk or clinical signs of mastitis. A milk sample was taken on the day of diagnosis and frozen for storage at $-20^{\circ} \mathrm{C}$. Samples were submitted to 1 of 4 bacteriology laboratories and a standardized protocol based on the National Mastitis Council guidelines for bacteriology culture and species identification was followed. Data on the number of colonies and species isolated was made available through the CBMRN database and obtained for use in this study. All cases of mastitis in any quarter were considered, regardless of culture result (Thompson-Crispi and Mallard, 2012). A new case in the same quarter was defined if $14 \mathrm{~d}$ had passed from the previous case (Barkema et al., 1998). Cows were at risk for the entire 2-yr period of CBMRN National Cohort of Dairy Farms sampling. Days at risk for each cow were calculated as the total number of DIM from start to end of the 2-yr period based on information available through the CBMRN. Time at risk ended if the cow was removed from the herd or was not milking. The incidence rate of clinical mastitis (IRCM) was calculated as the number of cases of mastitis for each cow per 36,500 d at risk (100 cow-years; Thompson-Crispi et al., 2013). Clinical mastitis was also calculated as a binary trait within the lactation of immune response sampling to evaluate associations with NAb.

\section{Statistical Analysis}

Complete records for $\mathrm{NAb}, \mathrm{SpAb}$, and cow registration numbers were available for 451 cows. The full pedigree records file included a total of 29,336 animals tracing back to 1883 and was provided by the Canadian Dairy Network (Guelph, Ontario). Antibody response at $\mathrm{d} 0$ was fit as a covariate for SpAb. Antibody was analyzed using a series of uni- and tri-variate linear animal models as follows:

$$
\begin{aligned}
y_{i j k l}=\mu_{i j k l} & +h t_{i}+p_{j}+\alpha \times s t+\beta \times s t^{2} \\
& +\gamma \times d_{k}+c_{l}+e_{i j k l},
\end{aligned}
$$

where $y_{i j k l}=$ NAb IgG, NAb IgM, or SpAb IgG1; $\mu_{i j k l}$ $=$ the overall mean; $h t_{i}=$ the random effect of herdtechnician ( 41 herds and 9 technicians); $p_{j}=$ the fixed effect of parity $(1,2,3$, and $\geq 4)$; st $=$ the stage of lactation measured as days in milk as a linear effect and $s t^{2}=$ stage of lactation squared; $d_{k}=\mathrm{d} 0$ data for $\mathrm{SpAb}$ as a fixed regression and $\alpha, \beta$, and $\gamma$ were regression coefficients; $c_{l}=$ the random effect of cow; and $e_{i j k l}=$ the residual error. The Shapiro-Wilk test statistic was used to test normality. Natural IgG antibody was logtransformed to normalize the distribution.

Heritability values were calculated as

$$
h^{2}=\sigma_{a}^{2} /\left(\sigma_{a}^{2}+\sigma_{h t}^{2}+\sigma_{e}^{2}\right),
$$

where $\sigma_{a}^{2}=$ additive genetic variance; $\sigma_{h t}^{2}=$ herd-technician variance; and $\sigma_{e}^{2}=$ residual variance. The genetic analysis was performed with the DMU package using an average information-REML algorithm for estimation of co(variance) components in mixed models (Madsen and Jensen, 2008).

Genetic parameters obtained from the linear animal model were used to estimate breeding values using the same model as above. Cows were categorized as high, average, or low immune responders if their antibody EBV was $>1$, between 1 and -1 , or $<-1$ standard deviation from the mean, respectively, for each NAb antibody trait. The association of the IRCM with immune response category was analyzed by Poisson regression using PROC GLIMMIX (SAS version 9.1.3; SAS Institute Inc., Cary, NC) with the natural logarithm of the number of days at risk as the offset and herd as a random effect. Least squares means were estimated and 
Table 1. Descriptive statistics of natural $(\mathrm{NAb})$ and specific $(\mathrm{SpAb})$ antibody of Canadian Holstein dairy cows $(\mathrm{n}=451)$

\begin{tabular}{|c|c|c|c|c|c|c|c|c|}
\hline Antibody & Antigen & Day $^{1}$ & Unit $^{2}$ & No. & Mean & SD & Minimum & Maximum \\
\hline NAb IgG & $\mathrm{KLH}^{3}$ & 0 & OD & 451 & 59.72 & 50.94 & 3.114 & 354.9 \\
\hline NAb IgM & KLH & 0 & OD & 451 & 112.4 & 30.54 & 40.30 & 239.4 \\
\hline SpAb IgG1 ${ }^{4}$ & $\mathrm{HEWL}^{5}$ & 14 & OD & 451 & 65.03 & 51.62 & 2.380 & 201.9 \\
\hline
\end{tabular}

${ }^{1}$ Day in the immunization protocol.

${ }^{2} \mathrm{OD}=$ optical density units $\times 10^{2}$ at $405 \mathrm{~nm}$.

${ }^{3}$ Keyhole limpet hemocyanin.

${ }^{4}$ Data published previously (Thompson-Crispi et al., 2012b).

${ }^{5}$ Hen egg white lysozyme.

Tukey's test was used to compare differences between high, average, and low responders, as reported previously for cows classified based on SpAb (ThompsonCrispi et al., 2013). Associations of mastitis with NAb were also evaluated with a logistic regression using PROC LOGISITIC.

\section{RESULTS}

Descriptive statistics for $\mathrm{NAb}$ and $\mathrm{SpAb}$ are presented in Table 1. Natural antibody to KLH of both the $\operatorname{IgG}$ and IgM isotypes were measureable in this study. Table 2 shows the estimates of genetic, herd-technician, residual, and total variances, as well as heritability estimates from the single trait analysis. For all traits, the additive genetic variance was a larger component of the total variance compared with herd-technician variances, which was not unexpected, as technicians were trained with a standard protocol of testing. The heritability of NAb of the IgG isotype was 0.32 ( SE $=0.11)$ and of the NAb IgM isotype was $0.18(\mathrm{SE}=$ $0.10)$. The heritability for $\mathrm{SpAb}$ was $0.27(\mathrm{SE}=0.09)$, as reported previously (Thompson-Crispi et al., 2012b).

Estimates of heritability, as well as genetic and residual correlations, are shown in Table 3. These heritability estimates are from a tri-variate analysis and are in agreement with the heritability found from the single trait analysis shown in Table 2 . The heritability estimates from the tri-variate analysis were $0.31(\mathrm{SE}=$
$0.11)$ for $\mathrm{NAb} \operatorname{IgG}, 0.16(\mathrm{SE}=0.11)$ for $\mathrm{NAb} \operatorname{IgM}$ and $0.24(\mathrm{SE}=0.10)$ for SpAb. No significant genetic correlations were found both within $\mathrm{NAb}$ ( $\operatorname{IgG}$ and $\operatorname{IgM}$ ) and between NAb and SpAb. However, the correlation between NAb and SpAb tended to be negative, -0.33 $(\mathrm{SE}=0.44)$ and $-0.07(\mathrm{SE}=0.33)$, for $\operatorname{IgM}$ and $\operatorname{IgG}$, respectively. The correlation between $\mathrm{NAb} \operatorname{IgG}$ and IgM was also negative, $-0.41(\mathrm{SE}=0.38)$.

Cows were classified as high, average, or low immune responders based on estimated breeding values for NAb binding to KLH. No significant differences in the IRCM per 100 cow-years for cows categorized by NAb IgG were found (Table 4), as demonstrated previously for these cows when categorized based on SpAb (Thompson-Crispi et al., 2013). However, the high-NAb IgM cows tended to have a lower IRCM, 19.0 cases per 100-cow years, compared with average- $(31.2$ cases per 100 -cow years; $P=0.082)$ and low- $(26.3$ cases per 100-cow years; $P=0.095)$ NAb IgM cows. The logistic regression analysis also showed increasing IgM binding to KLH to be associated with a decreased risk of clinical mastitis (odds ratio of 0.958; confidence interval 0.927-0.990). No association was found with NAb IgG.

\section{DISCUSSION}

Natural antibody binding to KLH, in the absence of prior immunization, was present in the serum of

Table 2. Additive genetic variance $\left(\sigma_{a}^{2}\right)$, herd-technician variance $\left(\sigma_{h t}^{2}\right)$, residual variance $\left(\sigma_{e}^{2}\right)$, total variance, and heritability $\left(\mathrm{h}^{2}\right)$ from a single trait analysis (SE in parentheses) of natural (NAb) and specific (SpAb) antibody of Canadian Holstein dairy cows $(\mathrm{n}=451)$

\begin{tabular}{lllllllll}
\hline Trait & Antigen & Day $^{1}$ & Unit $^{2}$ & $\sigma_{a}^{2}$ & $\sigma_{h t}^{2}$ & $\sigma_{e}^{2}$ & Total & $\mathrm{h}^{2}$ \\
\hline NAb IgG & KLH $^{3}$ & 0 & OD & 514 & 21.9 & 1,060 & 1,590 & $0.32(0.11)$ \\
NAb IgM & KLH $^{2}$ & 0 & OD & 158 & 64.0 & 668 & 890 & $0.18(0.10)$ \\
SpAb IgG1 & HEWL $^{5}$ & 14 & OD & 668 & 252 & 1,560 & 2,480 & $0.27(0.09)$ \\
\hline
\end{tabular}

${ }^{1}$ Day in the immunization protocol.

${ }^{2} \mathrm{OD}=$ optical density units $\times 10^{2}$ at $405 \mathrm{~nm}$.

${ }^{3}$ Keyhole limpet hemocyanin.

${ }^{4}$ Data published previously (Thompson-Crispi et al., 2012b).

${ }^{5}$ Hen egg white lysozyme. 
Table 3. Average of heritability (diagonal), genetic (above diagonal), and residual (below diagonal) correlations of natural (NAb) and specific (SpAb) antibody ( $\mathrm{SE}$ in parentheses) of $\mathrm{NAb}$ and $\mathrm{SpAb}$ of Canadian Holstein dairy cows $(\mathrm{n}=451)$

\begin{tabular}{llcc}
\hline Antibody & NAb IgG & NAb IgM & SpAb IgG1 \\
\hline NAb IgG & $\mathbf{0 . 3 2 ( 0 . 1 1 )}$ & $-0.41(0.38)$ & $0.07(0.33)$ \\
NAb IgM & $0.17(0.13)$ & $\mathbf{0 . 1 6}(\mathbf{0 . 0 9})$ & $-0.33(0.45)$ \\
SpAb IgG1 & $0.01(0.14)$ & $0.12(0.12)$ & $\mathbf{0 . 2 4}(\mathbf{0 . 1 0})$ \\
\hline
\end{tabular}

the Canadian Holstein dairy cows in this study, as demonstrated previously in Dutch Holstein-Friesians (van Knegsel et al., 2007; Ploegaert et al., 2011). The heritability estimates for NAb IgG found here are similar to a previous study that estimated an across herd heritability of NAb-binding KLH of 0.36 (Ploegaert et al., 2010). In that study, total antibody titers were measured in milk rather than the specific IgG and IgM isotypes measured in serum in the present study. Previous work has demonstrated NAb-binding KLH in milk and serum samples of dairy cows to be highly correlated (0.7), although titers of NAb in serum were consistently higher than milk samples (Ploegaert et al., 2011).

No significant genetic correlations were found between $\mathrm{NAb}$ of the IgG or IgM isotypes, nor with $\mathrm{SpAb}$ in this study. These results differed from a previous study in Holsteins that found correlations ranging from 0.45 to 0.99 for NAb-binding LPS, lipoteichoic acid (LTA), and peptidoglycan (PGN) and KLH (Ploegaert et al., 2010). It should be noted that the SpAb was measured to a different test antigen than NAb, which was measured to KLH in the current study. Future studies immunizing cows with KLH to determine the genetic correlations between NAb and SpAb to the same antigen may shed more light on this question.

The number of animals used in the current study to estimate genetic parameters was relatively small, which likely contributed to the rather large standard errors. Further, this study was performed as part of a larger study by the CBMRN with the goal to evaluate mastitis, and pedigree structure was not prioritized for a genetic study. Although data size and structure is limited, this study provides novel insight into the correlation of natural and specific antibodies in Canadian Holstein cows, as well as the correlation of NAb with mastitis. A follow-up study with a larger sample would improve the analysis by estimating the genetic relationship between clinical mastitis with NAb, which was not feasible in the current study due to the limited sample size.

No differences in the IRCM were found when cows were classified as high, average, or low for NAb $\operatorname{IgG}$. However, the high-NAb IgM cows had a tendency to have the lowest IRCM, 19.0 cases per 100 cow-years, compared with average and low responders (31.2 and
Table 4. Incidence rate of clinical mastitis per 100 cow-years by high, average, or low immune response category based on estimated breeding values for natural antibodies (NAb) IgM and $\operatorname{IgG}$ for Canadian Holstein cows $(\mathrm{n}=451)$

\begin{tabular}{lccc}
\hline Item & High & Average & Low \\
\hline NAb IgM & 19.0 & 31.2 & 26.3 \\
NAb IgG & 32.3 & 24.1 & 30.9 \\
\hline
\end{tabular}

26.3, respectively). Cows in the current study were ranked previously based on SpAb responses, and highimmune response cows had a significantly less mastitis, 17.1 cases per 100-cows years, compared with average and low responders (27.9 and 30.1, respectively; Thompson-Crispi et al., 2013). Previous work has also found cows with superior SpAb responses to have lower occurrence of other diseases, including mastitis, metritis, retained placenta, and displaced abomasum (Thompson-Crispi et al., 2012a). Therefore, breeding for enhanced SpAb responses is expected to improve inherent animal health and resistance to a multitude of diseases.

When mastitis was analyzed as a binary trait within the lactation of immune response sampling, NAb IgM was associated with a decreased risk for clinical mastitis $(\mathrm{OR}=0.958)$. Although the decreased risk is small, results suggest NAb IgM may be an additional tool to select for mastitis resistance in dairy cattle. The ICRM may be a more accurate reflection of the mastitis situation on Canadian dairy farms, given that it accounts for multiple cases per cow over the 2-yr study period.

Adaptive immune responses are critical to provide protection against pathogens that escape the innate immune system. Cell-mediated and antibody-mediated immune responses predominate in control against intra- and extracellular pathogens respectively, and both are required for broad-based disease resistance. Future studies should determine if NAb $\operatorname{IgM}$ is associated with a decreased risk of other diseases, as has been shown for SpAb (Thompson-Crispi et al., 2012a). Selection for resistance to a particular disease, such as mastitis, can have negative consequences for protection against other diseases. Antibody-mediated immune responses are negatively genetically correlated with CMIR (Thompson-Crispi et al., 2012b), and selection for mastitis resistance, typically controlled by antibody responses, may inadvertently lead to decreased cell-mediated immunocompetence, which is critical for protection against intracellular pathogens such as MAP (Pinedo et al. 2009). It is important to consider these genetic aspects of immune responses when designing a breeding program to improve animal health.

Studies in dairy cattle have evaluated NAb to bacterial components such as LPS, LTA, and PGN (Ploegaert 
et al., 2010; van Knegsel et al., 2012), which are common in the environment and host microflora. It is debatable if antibodies binding environmental antigens are truly NAb. Variation in animal exposure, as well as previous and concurrent infections, would likely influence antibody titers to these antigens, as these antigens have been shown to have immunomodulatory effects (Parmentier et al., 2004b). It has been suggested that, in the absence of intentional challenge and if animals are housed in the same environment, antibody binding common environmental antigens can be defined as NAb (Star et al., 2007). However, the latter was performed on chickens housed in the same facility and fed the same diet, which likely is not the case on large, national studies in dairy cattle. Nonetheless, in a study performed by Ploegaert et al. (2010) evaluating potential parameters for natural resistance in dairy cattle, IgG1-binding LTA was associated with an increased risk for clinical mastitis $(\mathrm{OR}=1.18)$. No significant associations were found for KLH antibody and incidence of clinical mastitis, as reported here, but NAb of the IgG1 isotype binding $\mathrm{KLH}$ was associated with a decreased the risk of a high SCC (>150,000 cells/mL) (Ploegaert, 2010).

Limited work has been done to evaluate NAb and disease in dairy cattle; however, a recent study by van Knegsel et al. (2012) evaluated the correlation of NAb and NAb to autoantigens $(\mathbf{N}(\mathbf{A}) \mathbf{A b})$ with metabolic and mammary health. Natural antibodies in serum binding KLH and LPS tended to be negatively correlated with NEFA concentration; paradoxically, the same NAb in milk were positively associated with NEFA concentration (van Knegsel et al., 2012), as demonstrated previously (van Knegsel et al., 2007). As excessive circulating levels of NEFA are associated with decreased bovine immune cell functionality (Ster et al., 2012) and increased disease incidence (Sordillo et al., 2009), the authors suggest a relationship between metabolic health and NAb. Natural autoantibodiesbinding transferrin in milk tended $(P<0.10)$ to be lower, and $\mathrm{N}(\mathrm{A}) \mathrm{Ab}$ to myosin tended to be higher in cows that had clinical mastitis when compared with healthy cows with low or high somatic cell scores (van Knegsel et al., 2012). This was the first study to evaluate $\mathrm{N}(\mathrm{A}) \mathrm{Ab}$ in cattle and the relationship with health, and the authors concluded further research was needed to determine the predictive value of $\mathrm{N}(\mathrm{A}) \mathrm{Ab}$ for udder health. Although $\mathrm{N}(\mathrm{A}) \mathrm{Ab}$ are important in the maintenance of homeostasis (Poletaev and Osipenko, 2003), selection for enhanced titers of antibody-binding self-antigens may have potentially deleterious effects in the long term. Antibody-binding self-antigens have been associated with miscarriage (Sater et al., 2011), and are responsible for autoimmune diseases in humans with severe immunopathology, such as systemic lupus erythematosus (Ching et al., 2012) and multiple sclerosis (Mayer and Meinl, 2012). Distinguishing between $\mathrm{NAb}$ responsible for maintenance of homeostasis and conventional antibody-binding self-antigens, which is generally associated with autoimmunity, would be difficult if not impossible.

More extensive selective breeding experiments for NAb have been performed in other species, such as chicken. Survival experiments found low levels of NAbbinding KLH or high levels of NAb-binding LPS in chickens that did not survive the laying period (Star et al., 2007). Experiments evaluating the correlation between SpAb and NAb found that chickens divergently selected for high specific antibody responses to sheep red blood cells had significantly higher levels of NAbbinding ovalbumin and KLH (Parmentier et al., 2004a). Genetic parameters of NAb and SpAb in those chickens were subsequently measured, and a weak, though positive, correlation was found (Wijga et al., 2009). The authors suggest that selection for enhanced adaptive immune responses may result in a positive correlation with innate immunity in chickens (Wijga et al., 2009). The beneficial aspects of NAb found in the current study and in other species further warrant future studies investigating associations of NAb with $\mathrm{SpAb}$ and disease in dairy cattle.

Natural antibodies provide a link between the innate and adaptive immune systems via enhanced antigen uptake and processing, as well as presentation to B cells or dendritic cells, as reviewed by Ochsenbein and Zinkernagel (2000). Natural antibodies can prevent pathogen dissemination to vital organs by improving immunogenicity through enhanced antigen trapping in secondary lymphoid organs (Ochsenbein et al., 1999), and have been shown to mediate dendritic cell migration, thereby shaping the generation of adaptive immune responses (Rapaka et al., 2010). Appropriate innate host defenses, such as NAb, are critical for signaling adaptive responses for the resolution of infection (Lippolis, 2008). If a positive genetic correlation were to exist in cattle between NAb and SpAb, a selection approach for robust adaptive immune responses may result in positive correlations with at least certain innate host defense mechanisms, as suggested by Wijga et al. (2009). However, results of this study found no genetic correlation between $\mathrm{NAb}$ and $\mathrm{SpAb}$, suggesting that $\mathrm{NAb}$ could be used as an additional tool to select for mastitis resistance. As Holstein dairy cows with superior specific antibody responses have a lower occurrence of many diseases compared with other cows (Thompson-Crispi et al., 2012a), and adaptive immune responses are critical to provide protection against anti- 
gens that escape the innate immune system, AMIR and CMIR should not be overlooked in breeding programs to improve animal health.

\section{CONCLUSIONS}

The results of this study indicate that, in Canadian Holstein cows, natural antibody of the IgM isotype was associated with a decreased risk for clinical mastitis; however, NAb IgG was not. Cows classified as high for NAb IgM tended to have a lower incidence rate of clinical mastitis compared with other cows. Natural antibodies may provide an additional tool to select for mastitis resistance, however specific CMIR and AMIR, which are critical to provide protection against a variety of intracellular and extracellular organisms, respectively, should not be overlooked in a selection strategy for broad-based disease resistance. No significant genetic correlations were observed between the NAb and $\mathrm{SpAb}$ response to a test antigen. Given the relatively small sample size, the correlation between $\mathrm{NAb}$ and $\mathrm{SpAb}$ and the association of $\mathrm{NAb} \operatorname{IgM}$ and clinical mastitis should be investigated in future studies due to beneficial associations found here and in other species.

\section{ACKNOWLEDGMENTS}

This research was financed by grants to B. A. Mallard from National Sciences and Engineering Research Council of Canada; Alberta Milk; Dairy Farmers of New Brunswick, Nova Scotia, Ontario, and Prince Edward Island; Novalait Inc.; Dairy Farmers of Canada; DairyGen council of Canadian Dairy Network; Agriculture and Agri-Food Canada; Public Health Agency of Canada; Technology PEI Inc.; Université de Montréal; and University of Prince Edward Island through the Canadian Bovine Mastitis Research Network. K. A. Thompson-Crispi was funded by the Dairy Farmers of Ontario Doctoral Research Assistantship. The authors thank Astrid Koeck (University of Guelph) for help in analyzing the data and Douglas Hodgins (University of Guelph) for technical advice.

\section{REFERENCES}

Barkema, H. W., Y. H. Schukken, T. J. Lam, M. L. Beiboer, H. Wilmink, G. Benedictus, and A. Brand. 1998. Incidence of clinical mastitis in dairy herds grouped in three categories by bulk milk somatic cell counts. J. Dairy Sci. 81:411-419.

Baumgarth, N. 2011. The double life of a B-1 cell: Self-reactivity selects for protective effector functions. Nat. Rev. Immunol. 11:34-46.

Baumgarth, N., J. W. Tung, and L. A. Herzenberg. 2005. Inherent specificities in natural antibodies: A key to immune defense against pathogen invasion. Springer Semin. Immunopathol. 26:347-362.

Boes, M. 2000. Role of natural and immune IgM antibodies in immune responses. Mol. Immunol. 37:1141-1149.
Ching, K. H., P. D. Burbelo, C. Tipton, C. Wei, M. Petri, I. Sanz, and M. J. Iadarola. 2012. Two major autoantibody clusters in systemic lupus erythematosus. PLoS ONE 7:e32001.

Harris, J. R., and J. Markl. 1999. Keyhole limpet hemocyanin (KLH): A biomedical review. Micron 30:597-623.

Heriazon, A., K. A. Thompson, B. N. Wilkie, W. Mathes-Sears, M. Quinton, and B. A. Mallard. 2009. Antibody to ovalbumin and delayed-type hypersensitivity to Candida albicans and mycobacteria in lactating Holstein cows using Quil A or Freund's complete adjuvant. Vet. Immunol. Immunopathol. 127:220-227.

Kohler, H., J. Bayry, A. Nicoletti, and S. V. Kaveri. 2003. Natural autoantibodies as tools to predict the outcome of immune response? Scand. J. Immunol. 58:285-289.

Lippolis, J. D. 2008. Immunological signaling networks: Integrating the body's immune response. J. Anim. Sci. 86(E-Suppl.):E53E63.

Madsen, P., and J. Jensen. 2008. DMU: A user's guide. A package for analysing multivariate mixed models. DJF, Foulum, Denmark.

Mallard, B. A., H. Atalla, S. Cartwright, B. C. Hine, B. Hussey, M. Paibomesai, K. A. Thompson-Crispi, and L. Wagter-Lesperance. 2011. Genetic and epigenetic regulation of the bovine immune system: Practical implications of the high immune response technology. Proc. Natl. Mastitis Counc. 50th Annu. Mtg. (1):53-63. National Mastitis Council, Arlington, VA.

Mallard, B. A., L. C. Wagter, M. J. Ireland, and J. C. Dekkers. 1997. Effects of growth hormone, insulin-like growth factor-I, and cortisol on periparturient antibody response profiles of dairy cattle. Vet. Immunol. Immunopathol. 60:61-76.

Mayer, M. C., and E. Meinl. 2012. Glycoproteins as targets of autoantibodies in CNS inflammation: MOG and more. Ther. Adv. Neurol. Disord. 5:147-159.

Ochsenbein, A. F., T. Fehr, C. Lutz, M. Suter, F. Brombacher, H. Hengartner, and R. M. Zinkernagel. 1999. Control of early viral and bacterial distribution and disease by natural antibodies. Science 286:2156-2159.

Ochsenbein, A. F., and R. M. Zinkernagel. 2000. Natural antibodies and complement link innate and acquired immunity. Immunol. Today 21:624-630.

Parmentier, H. K., A. Lammers, J. J. Hoekman, R. G. De Vries, I. T. Zaanen, and H. F. Savelkoul. 2004a. Different levels of natural antibodies in chickens divergently selected for specific antibody responses. Dev. Comp. Immunol. 28:39-49.

Parmentier, H. K., W. J. Van Den Kieboom, M. G. Nieuwland, R. G. De Vries, B. N. Hangalapura, H. F. Savelkoul, and A. Lammers. 2004b. Differential effects of lipopolysaccharide and lipoteichoic acid on the primary antibody response to keyhole limpet hemocyanin of chickens selected for high or low antibody responses to sheep red blood cells. Poult. Sci. 83:1133-1139.

Pinedo, P. J., A. Donovan, O. Rae, and J. DeLapaz. 2009. Association between paratuberculosis infection and general immune status in dairy cattle. Page 127 in Proc. 10th Int. Colloq. Paratuberculosis. International Association for Paratuberculosis, Minneapolis, MN.

Ploegaert, T. C. 2010. Parameters for natural resistance in bovine milk. PhD Thesis. Wageningen University, Wageningen, the Netherlands.

Ploegaert, T. C., E. Tijhaar, T. J. Lam, A. Taverne-Thiele, J. J. van der Poel, J. A. van Arendonk, H. F. Savelkoul, and H. K. Parmentier. 2011. Natural antibodies in bovine milk and blood plasma: Variability among cows, repeatability within cows, and relation between milk and plasma titers. Vet. Immunol. Immunopathol. 144:88-94.

Ploegaert, T. C., S. Wijga, E. Tijhaar, J. J. van der Poel, T. J. Lam, H. F. Savelkoul, H. K. Parmentier, and J. A. van Arendonk. 2010. Genetic variation of natural antibodies in milk of Dutch HolsteinFriesian cows. J. Dairy Sci. 93:5467-5473.

Poletaev, A., and L. Osipenko. 2003. General network of natural autoantibodies as immunological homunculus (Immunculus). Autoimmun. Rev. 2:264-271.

Rapaka, R. R., D. M. Ricks, J. F. Alcorn, K. Chen, S. A. Khader, M. Zheng, S. Plevy, E. Bengten, and J. K. Kolls. 2010. Conserved natural IgM antibodies mediate innate and adaptive immunity 
against the opportunistic fungus Pneumocystis murina. J. Exp. Med. 207:2907-2919.

Reyher, K. K., S. Dufour, H. W. Barkema, C. L. Des, T. J. Devries, I. R. Dohoo, G. P. Keefe, J. P. Roy, and D. T. Scholl. 2011. The National Cohort of Dairy Farms - A data collection platform for mastitis research in Canada. J. Dairy Sci. 94:1616-1626.

Sater, M. S., R. R. Finan, F. E. Mustafa, G. M. Al-Khateeb, and W. Y. Almawi. 2011. Anti-annexin V IgM and IgG autoantibodies and the risk of idiopathic recurrent spontaneous miscarriage. J. Reprod. Immunol. 89:78-83.

Sordillo, L. M., G. A. Contreras, and S. L. Aitken. 2009. Metabolic factors affecting the inflammatory response of periparturient dairy cows. Anim. Health Res. Rev. 10:53-63.

Star, L., K. Frankena, B. Kemp, M. G. Nieuwland, and H. K. Parmentier. 2007. Natural humoral immune competence and survival in layers. Poult. Sci. 86:1090-1099.

Ster, C., M. C. Loiselle, and P. Lacasse. 2012. Effect of postcalving serum nonesterified fatty acids concentration on the functionality of bovine immune cells. J. Dairy Sci. 95:708-717.

Thompson-Crispi, K. A., B. Hine, M. Quinton, F. Miglior, and B. A. Mallard. 2012a. Short communication: Association of disease incidence and adaptive immune response in Holstein dairy cows. J. Dairy Sci. 95:3888-3893.

Thompson-Crispi, K. A., and B. A. Mallard. 2012. Type 1 and type 2 immune response profiles of commercial dairy cows in four regions of Canada. Can. J. Vet. Res. 76:120-128.
Thompson-Crispi, K. A., F. Miglior, and B. A. Mallard. 2013. Incidence rates of clinical mastitis among Canadian Holsteins classified as high, average and low immune responders. Clin. Vaccine Immunol. 20:106-112.

Thompson-Crispi, K. A., A. Sewalem, F. Miglior, and B. Mallard. $2012 \mathrm{~b}$. Genetic parameters of adaptive immune response traits in Canadian Holsteins. J. Dairy Sci. 95:401-409.

van Knegsel, A. T., R. G. De Vries, S. Meulenberg, H. van den Brand, J. Dijkstra, B. Kemp, and H. K. Parmentier. 2007. Natural antibodies related to energy balance in early lactation dairy cows. J. Dairy Sci. 90:5490-5498.

van Knegsel, A. T., M. Hostens, R. G. De Vries, A. Lammers, B. Kemp, G. Opsomer, and H. K. Parmentier. 2012. Natural antibodies related to metabolic and mammary health in dairy cows. Prev. Vet. Med. 103:287-297.

Wagter, L. C., B. A. Mallard, B. N. Wilkie, K. E. Leslie, P. J. Boettcher, and J. C. Dekkers. 2000. A quantitative approach to classifying Holstein cows based on antibody responsiveness and its relationship to peripartum mastitis occurrence. J. Dairy Sci. 83:488-498.

Wijga, S., H. K. Parmentier, M. G. Nieuwland, and H. Bovenhuis. 2009. Genetic parameters for levels of natural antibodies in chicken lines divergently selected for specific antibody response. Poult. Sci. $88: 1805-1810$ 